\title{
Los acabados arquitectónicos del claustro del Templete de Bramante en Roma. Aproximación a su estudio mediante el sistema de ordenación del color Munsell
}

\author{
Isolina Díaz-Ramos
}

\begin{abstract}
Resumen: El estudio del color original que conforma los edificios o conjuntos patrimoniales es una tarea necesaria a fin de conocer las unidades estratigráficas que se han sucedido en la historia del bien, contribuyendo a definir los entornos históricos. Pese a que en nuestro país se han realizado una serie de trabajos sobre este sujeto, continúa siendo necesaria la incorporación de este tipo de estudios en trabajos de conservación del patrimonio edificado. Por ello, se han realizado una serie de lecturas cromáticas sobre los muros del claustro del Templete de Bramante en Roma empleando un atlas Munsell, a fin de determinar los estratos, secuencias y tonos aplicados a lo largo de los siglos. Los resultados muestran una paleta de tonalidad cálida compuesta por amarillos, verdes, ocres y rojos. Este trabajo pretende poner en valor y conservar los acabados arquitectónicos, tomando como referencia los muros del patio del Templete de Bramante.
\end{abstract}

Palabras clave: color, unidades estratigráficas, sistema Munsell, mortero, estrato pictórico, lectura cromática, acabados arquitectónicos, conservación y documentación

\section{The architectural finishes of the cloister of the Bramante Temple in Rome. An approach by the use of the Munsell colour system}

Abstract: The study of the exterior decorative layers on heritage buildings and sites is a necessary task to produce knowledge on the original stratigraphic units that have defined historical environments. Despite the fact that some works have been carried out around this subject in our country, it is still necessary to incorporate this kind of studies on the conservation works of built heritage. Therefore, a number of chromatic readings have been carried out on the walls of the cloister of Bramante Temple in Rome, in order to determine the layers, sequences and tones applied over the centuries by the use of a Munsell atlas. Results are a warm tone palette of yellows, greens, ochres, and reds. This work aims to asses and conserve architectural finishes, taking as an example the walls of the courtyard of the Bramante Temple.

Keyword: colour, stratigraphic units, Munsell System, lime mortar, painting stratigraphy, colour matching, architectural finishes, conservation and documentation

\section{Os acabamentos arquitetónicos do claustro do Templete de Bramante em Roma. Aproximação ao seu estudo usando o sistema de cores de Munsell}

Resumo: O estudo da cor original que compõe os edifícios ou conjuntos patrimoniais é uma tarefa necessária para conhecer as unidades estratigráficas que se sucederam na história do bem, ajudando a definir a envolvente histórica. Apesar de terem sido realizados no nosso país vários trabalhos sobre este assunto, continua a ser necessária a incorporação deste tipo de estudos nas obras de conservação do património edificado. Por esta razão, realizou-se uma série de leituras cromáticas nas paredes do claustro do Templete de Bramante em Roma usando um atlas Munsell, a fim de determinar as camadas, sequências e tons aplicados ao longo dos séculos. Os resultados mostram uma paleta de tons quentes composta de amarelos, verdes, ocre e vermelhos. Este trabalho visa valorizar e preservar os acabamentos arquitetónicos, tendo como referência as paredes do pátio do Templete de Bramante.

Palavras-chave: cor, unidades estratigráficas, sistema Munsell, argamassa, camada pictórica, leitura de cores, acabamentos arquitetónicos, conservação e documentação 


\section{Introducción}

Una de las obras cumbre del Renacimiento italiano, el Templete de Bramante, se sitúa dentro de un conjunto arquitectónico de interés compuesto por la iglesia de San Pietro, su claustro exterior que abriga el Templete, y el segundo claustro, edificado originariamente para acoger el convento de padres franciscanos que presidían la iglesia, sede de la actual Academia de España en Roma.

Entre los años 1480 y 1500 se realizó la construcción de la iglesia de San Pietro y su claustro. El Templete de Bramante llegaría pocos años después, estando concluido hacia 1505. La construcción del segundo claustro que conformaría el antiguo convento de los padres franciscanos fue finalizado hacia 1557 (Jiménez 2013 y Cantatore 2017).

Estos dos claustros se convirtieron, junto a la iglesia de San Pietro, en un lugar de peregrinación sagrada con indulgencia plenaria, dado que en este conjunto monumental se halla el lugar en el que según una antigua tradición cristiana se sitúa el martirio de san Pedro (Canalda 2019). Albergaba originariamente cincuenta y una pinturas murales ejecutadas al fresco que representan escenas de la vida de san Francisco de Asís. Las realizadas en el primer claustro, entre los años 1587 y 1588, se atribuyen al taller de Giovanni Lombardelli. Niccolò Circignani, más conocido como il Pomarancio, pintó los lunetos localizados en el segundo claustro entre 1587 y 1590. En la actualidad, se conservan treinta y siete pinturas murales, hallándose once de ellas en el primer claustro, y veintiséis en el antiguo monasterio (idem).

Mucho se ha escrito acerca del monumento y del sitio. Sin embargo, se conoce muy poco acerca de los morteros y estratos pictóricos localizados en el primer claustro, claustro exterior o patio del Templete [Figura 1]. Desafortunadamente, el estudio de los acabados históricos ${ }^{[1]}$ que presenta un edificio o monumento en el momento de su construcción es una tarea que aún no está muy extendida en nuestro territorio, siendo el color un aspecto fundamental a tener en cuenta en trabajos de documentación y conservación de bienes patrimoniales arquitectónicos.

Este artículo traerá a la luz los acabados arquitectónicos del patio del Templete de Bramante, conformados originariamente por una paleta de tonalidad cálida y contribuyendo con ello a la puesta en valor del color original como un elemento más a considerar en las prácticas de conservación y restauración del patrimonio edificado.

Tras la presentación de los objetivos, este texto se detendrá brevemente en los estudios previos de color acometidos en el claustro del Templete dentro de la sección "Situación actual del tema a estudiar". Además, se revisará una serie de trabajos que han estudiado

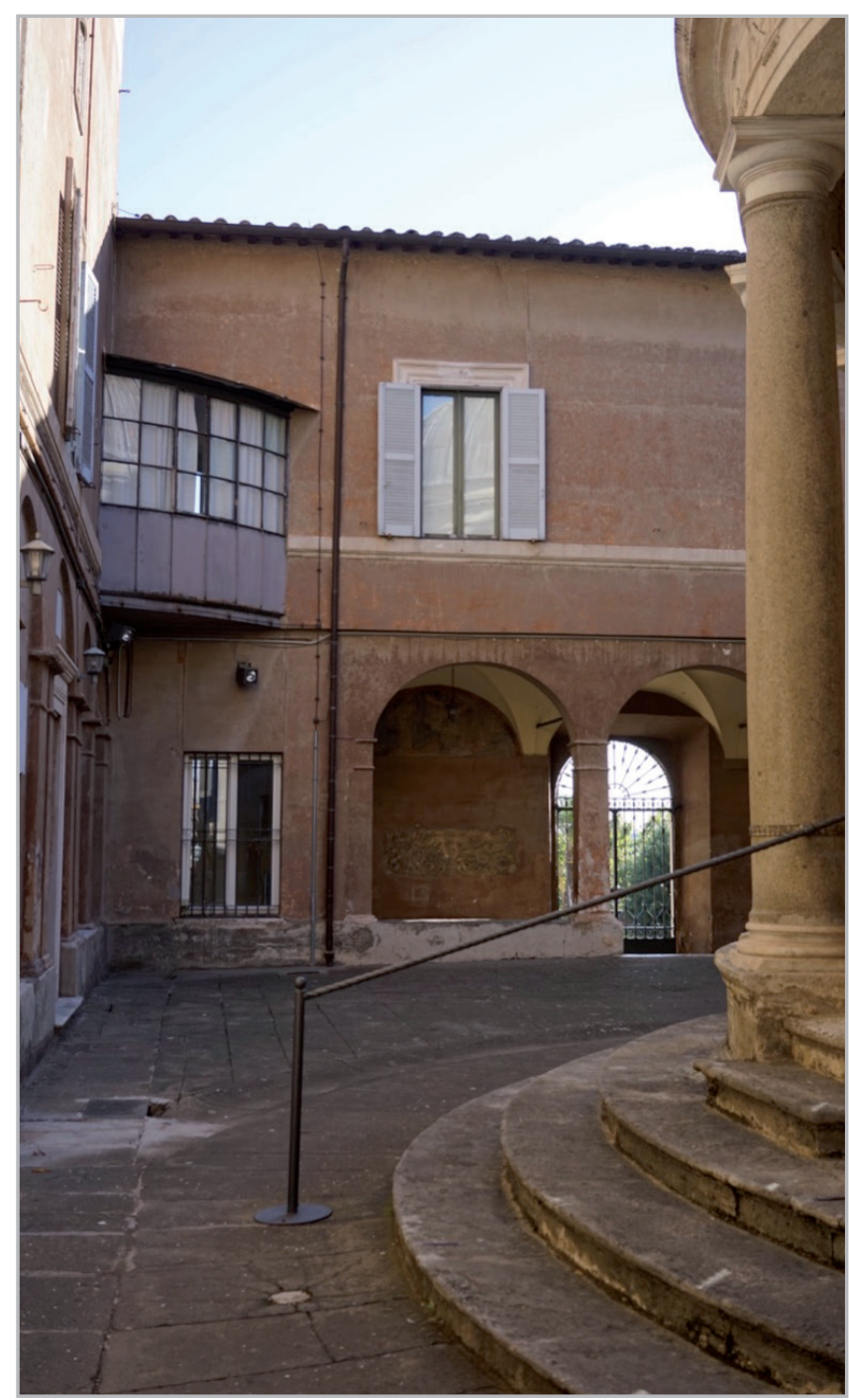

Figura 1.- Vista del Pórtico de Entrada desde el patio del Templete de Bramante. Créditos: Isolina Díaz-Ramos.

el color de determinadas ciudades o monumentos en nuestro país. Posteriormente, se presentará la metodología de estudio seguida en este trabajo, tras la que se introducirá de manera breve al sistema Munsell de ordenación de color. A partir de aquí, se presentarán las unidades estratigráficas documentadas en el Claustro del Templete, las concordancias de color y las posteriores conclusiones $^{[2] .}$

\section{Objetivos}

Los muros que componen el patio exterior del Templete nos ofrecen una lectura dispar en sus paños. La sucesión de reparaciones acometidas a lo largo de la historia, junto a las transformaciones efectuadas en el conjunto, afectan al valor patrimonial de autenticidad del sitio (International Council on Monuments and Sites 1994). La existencia de unidades estratigráficas en el claustro revela la ejecución anterior de estudios cromáticos, o un interés por conocer las capas de materia originales. 
A fin de iniciar una línea de trabajo que favorezca la puesta en valor y documentación de la imagen original y las sucesivas históricas que han tenido los muros que envuelven al Templete de Bramante, han sido formuladas una serie de preguntas que este documento intentará responder:

- ¿Qué acabados históricos componen los muros del claustro?

- ¿Qué color original presentaban las paredes del patio en el momento de su edificación?

- ¿Cuántos estratos pictóricos se han sucedido a lo largo de los siglos siguiendo los gustos estéticos de cada momento histórico?

El objetivo de este estudio es documentar y analizar los diferentes acabados históricos presentes en los muros del claustro del Templete de Bramante.

Esta documentación inicial pretende, además, servir como base sobre la que continuar investigando los originales históricos del primer claustro, así como contribuir y poner en valor la conservación de los acabados arquitectónicos en un sentido más generalista.

\section{Situación actual del tema a estudiar}

Como se ha indicado en la introducción, se ha encontrado en el claustro del Templete de Bramante una serie de unidades estratigráficas en diversas zonas de sus muros. Por ello, al inicio de esta investigación, se consultó en los archivos de la biblioteca de la Academia de España la documentación existente con el objeto de determinar quién y en qué momento pudo efectuar estas secuencias cromáticas. Se comenzó por el estudio de los documentos que hacían referencia al estado de conservación de la iglesia de San Pietro in Montorio, emitidos por Bosch, Roig, et al. en el año 2010, así como a la propuesta de intervención de las cubiertas del templo de San Pietro (Bosch 2014). Se pensó en un primer momento que las catas localizadas en el Muro Sur del claustro (FSa e ISa), situadas en la fábrica aledaña a la iglesia de San Pietro, podrían haber sido efectuadas por estos autores.

Previo a estos trabajos, Peláez realizó tareas de restauración de fábricas del claustro del Templete en el año 2003. Sin embargo, y al igual que ocurrió con los autores anteriores, no se encontró información acerca de estas catas. Finalmente fueron consultados, también sin éxito, otros textos sobre intervenciones efectuadas a lo largo de los años por Bueno (1985), Sancho Roda y Capponi (1998), Sancho Roda y Sánchez Barriga (19971999), Peláez (1992) y Peláez y Frechilla (2001, 2002a, 2002b).

En líneas generales, y tal y como se ha expresado al inicio de este artículo, el estudio y análisis de morteros y estratos pictóricos que presentan los edificios y monumentos históricos en su estado inicial es un tema poco tratado. Al contrario, continúa siendo una práctica general proceder al picado y posterior reposición de los morteros originales, sin apenas documentar o analizar los acabados arquitectónicos, por lo que poco se sabe del color original de numerosas obras arquitectónicas, menos aún de paisajes edificados.

En este sentido, resultan muy valiosas las aportaciones que han realizado desde comienzos de este siglo autores como García y Llopis (2010 y 2012), generando conocimiento en el color de ciudades mediterráneas como Ontiyent y Valencia, o las diferentes investigaciones en torno a los originales históricos de la ciudad de Granada efectuados por Collado, Medina y García (2004 y 2007). Estos autores se han apoyado en métodos científicos de análisis para llegar a conocer los acabados arquitectónicos de los edificios objeto de estudio.

De igual modo, Armenta realizó en el año 2014 estudios de color en la emblemática calle granadina del Darro, documentando las pinturas que adornan los muros de diferentes edificios. Similares aportaciones ofrecieron anteriormente Bermúdez-Coronel y Ruiz del Portal (2000) sobre el cromatismo de las puertas de Córdoba. Ambos trabajos siguen los modelos ejecutados por Giovanni Brino en la década de los ochenta del pasado siglo en diferentes ciudades italianas (1987, 1985a y 1985b). Todos ellos se basan en la toma de registros cromáticos basados en principios de percepción, es decir, con el apoyo de cartas y atlas de color. Sin embargo, la mayoría de ellos dejan fuera de su estudio los morteros y posibles capas de preparación que han de influenciar el color y la textura final.

\section{Método de trabajo y alcance del estudio}

Como se ha indicado, la presencia de unidades estratigráficas en el claustro exterior revela la ejecución anterior de un estudio cromático, o al menos un interés por conocer los diferentes estratos pictóricos que conforman el entorno del Templete de Bramante. Por ello, al inicio de esta investigación, se consultó en los archivos de la biblioteca de la Real Academia de España la documentación existente, a fin de localizar los informes efectuados sobre las catas presentes a día de hoy en el patio del Templete.

Posteriormente, durante la segunda quincena de febrero de 2019, se procedió a localizar y documentar las unidades estratigráficas que se hallaban en el claustro, efectuadas con toda probabilidad durante las diferentes intervenciones que ha tenido el conjunto arquitectónico. En este período, fueron realizadas dos nuevas catas de color a fin de complementar la totalidad de las lecturas tomadas.

Finalmente, en la última quincena de septiembre del mismo año, se completaron los trabajos de notación del 


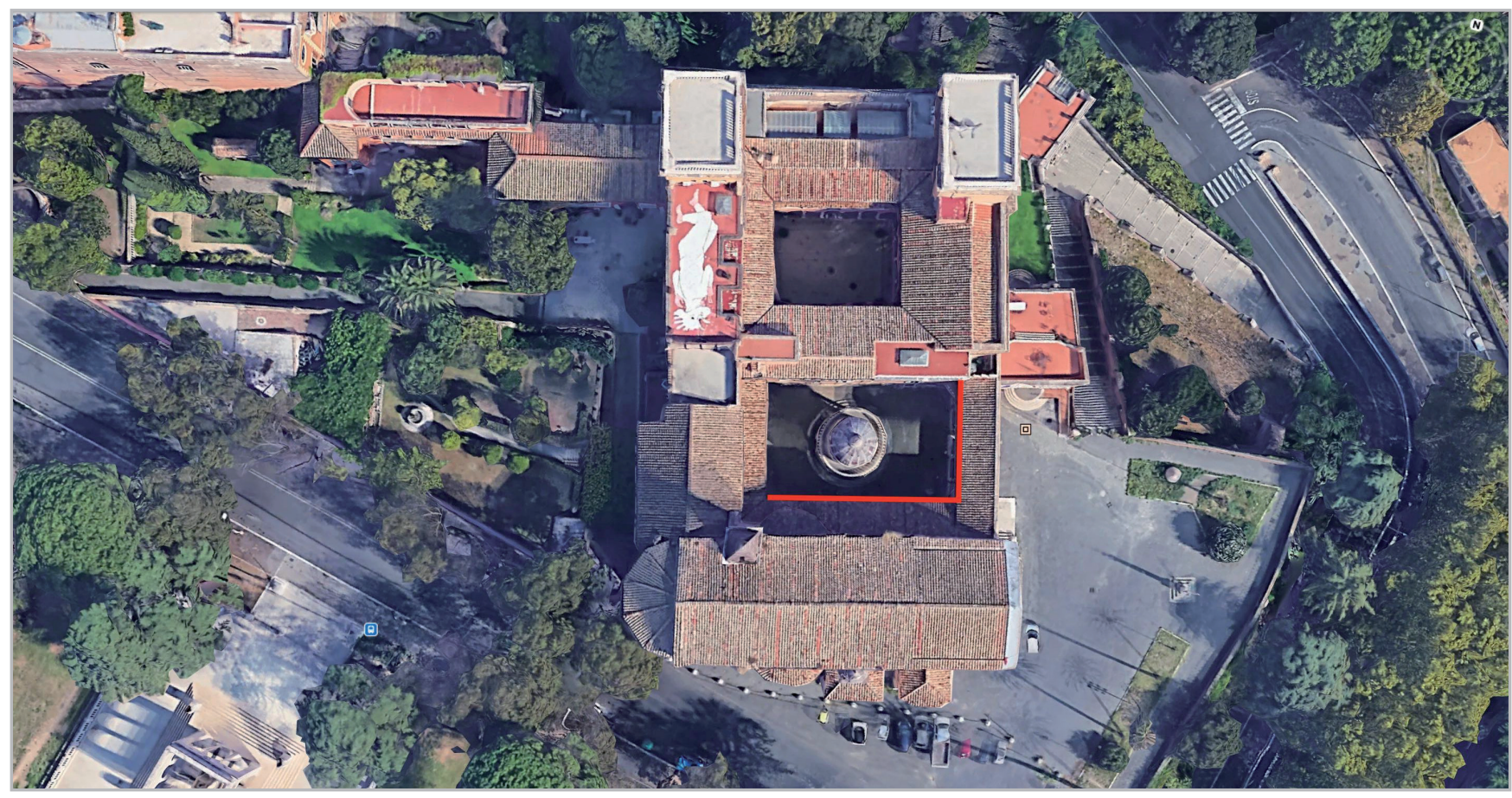

Figura 2.- Vista aérea del conjunto arquitectónico en el que se ha señalado en rojo la zona de estudio. Créditos: Google earth / Isolina Díaz-Ramos.

color de las unidades estratigráficas mediante el sistema Munsell. A su vez, se procedió a lecturas directas de diferentes estratos cromáticos sin necesidad de practicar raspados en los muros, aprovechando los desperfectos que éstos presentaban y que permitían determinar a simple vista las diferentes secuencias de color. Las lecturas fueron realizadas en los momentos en que la incidencia de rayos solares era mínima en el claustro, es decir, a primera hora de la mañana y última de la tarde.

En este estudio inicial se ha priorizado la lectura de aquellos paños que ya poseían unidades estratigráficas, por lo que los muros norte, este y oeste del claustro no han sido incluidos. Las paredes analizadas se encuentran en el Pórtico de Entrada al Templete por la plaza de San Pietro, también conocida como Galería Oriental (Canalda 2019), donde se hallaron un total de siete unidades estratigráficas, y en el Muro Sur, que contenía dos catas. Debido a la dificultad de lectura de las secuencia cromáticas de este paño, hubo que proceder a realizar dos unidades estratigráficas nuevas. Por lo tanto, a las nueve secciones cromáticas que se hallaban en estos espacios, se le sumaron dos más a fin de completar las lecturas. Los muros de estudio son, además, los que aún conservan la altura original del monumento, originalmente de dos plantas según muestran numerosos grabados de la época [Figura 2].

A su vez, una primera toma de muestras de acabados arquitectónicos se llevó a cabo en la zona de estudio, en aquellos lugares donde éstas se podían desprender con facilidad, como grietas y huecos presentes en las paredes.

\section{Nomenclatura empleada}

Los muros norte, sur y oeste del claustro de planta rectangular que envuelven al Templete de Bramante constan de un zócalo sobre el que se hallan dispuestas las basas de las pilastras de sección cuadrangular, sobre cuyo capitel se apoya una serie de arcadas de medio punto de huecos ciegos. Originariamente, estos arcos se encontraban abiertos hasta comienzos del siglo XVII, dando acceso a la iglesia de San Pietro en la panda meridional, y al convento franciscano por la galería norte (Bosch et al. 2010 y Canalda 2019).

La línea de imposta que decora el Muro Sur presenta en su superficie actual un revestimiento rugoso de tonalidades blanquecinas que imita la textura de un travertino, mientras que en los muros del patio, la última capa cromática emula un estuco veneciano. Estas capas superficiales son irregulares en la actualidad, debido a la presencia de repintes y parches de distintos materiales que se han aplicado durante sucesivas intervenciones.

A fin de sistematizar los datos tomados, éstos han sido designados de la siguiente forma: se nombra primero el lugar del muro donde se halla la secuencia, seguido del punto cardinal donde se sitúa el paño, y una letra minúscula. Ejemplo: ZSa (Z) Zócalo, (S) Sur, (a) letra de muestra [Figura 3].

Las catas de color ya presentes en el Pórtico de Entrada, se denominarán con una $\mathrm{E}$, seguida del punto cardinal y la letra de la muestra. Ejemplo: ENc. (E) Pórtico de Entrada, 


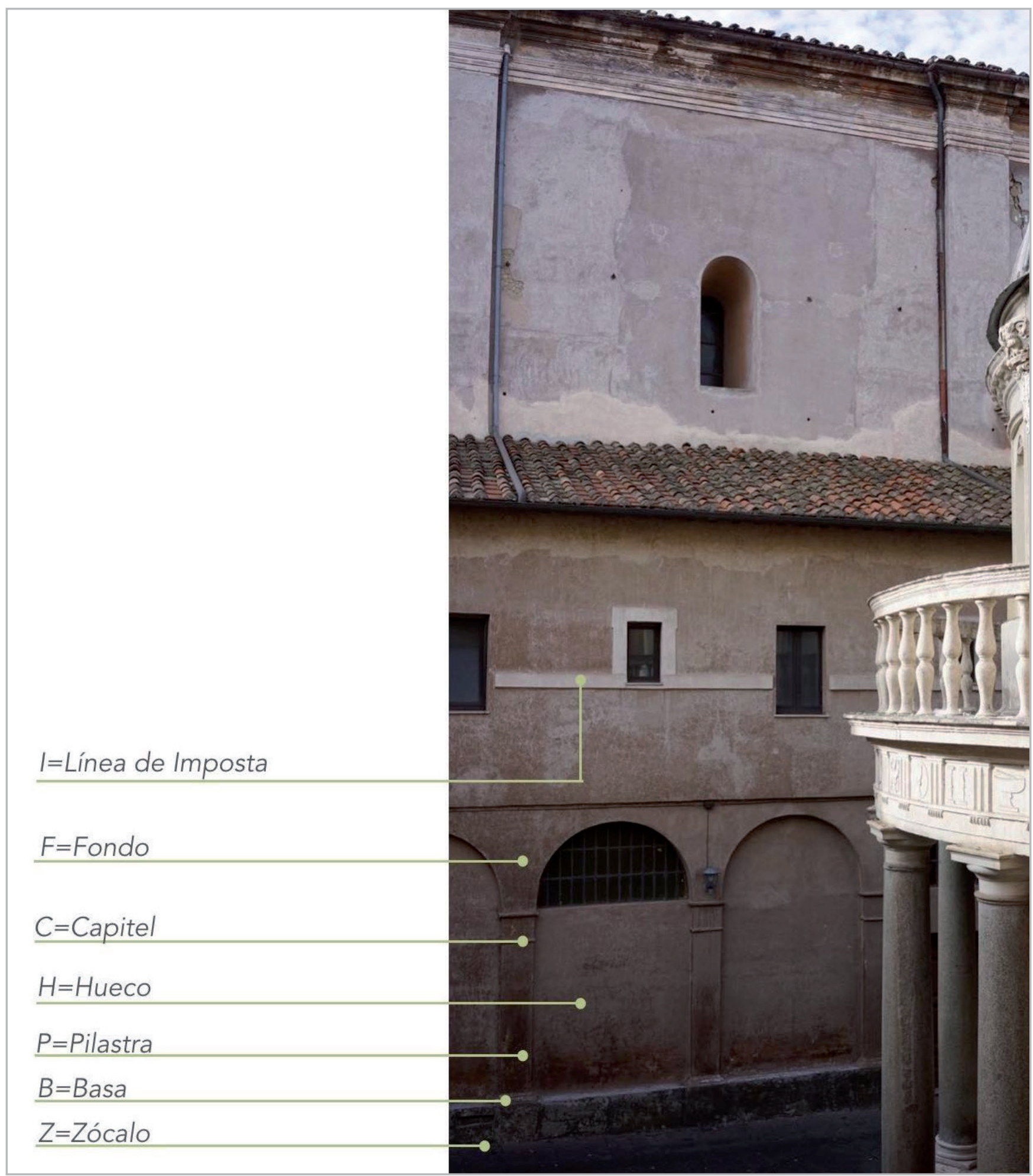

Figura 3.- Detalle del Muro Sur, sobre el que se muestra la nomenclatura empleada en este trabajo. Créditos: Isolina Díaz-Ramos.

(N) Norte, (c) letra de muestra.

Si bien las catas ya presentes en el sitio se han designado con una letra, el modo de nombrar las catas realizadas en el trabajo de documentación del año 2019 ha sido con un número. A modo de ejemplo tenemos EO1, donde $(E)$ se refiere al Pórtico de Entrada, $(\mathrm{O})$ oeste, y (1) número de la unidad estratigráfica efectuada por la autora.

En los alzados de los muros de estudio, se situaron junto a las catas que ya existían, las nuevas efectuadas (FS1 e IS1), y otras lecturas realizadas directamente sobre las paredes, es decir, donde no se procedió al raspado del muro [Figura 4]. 

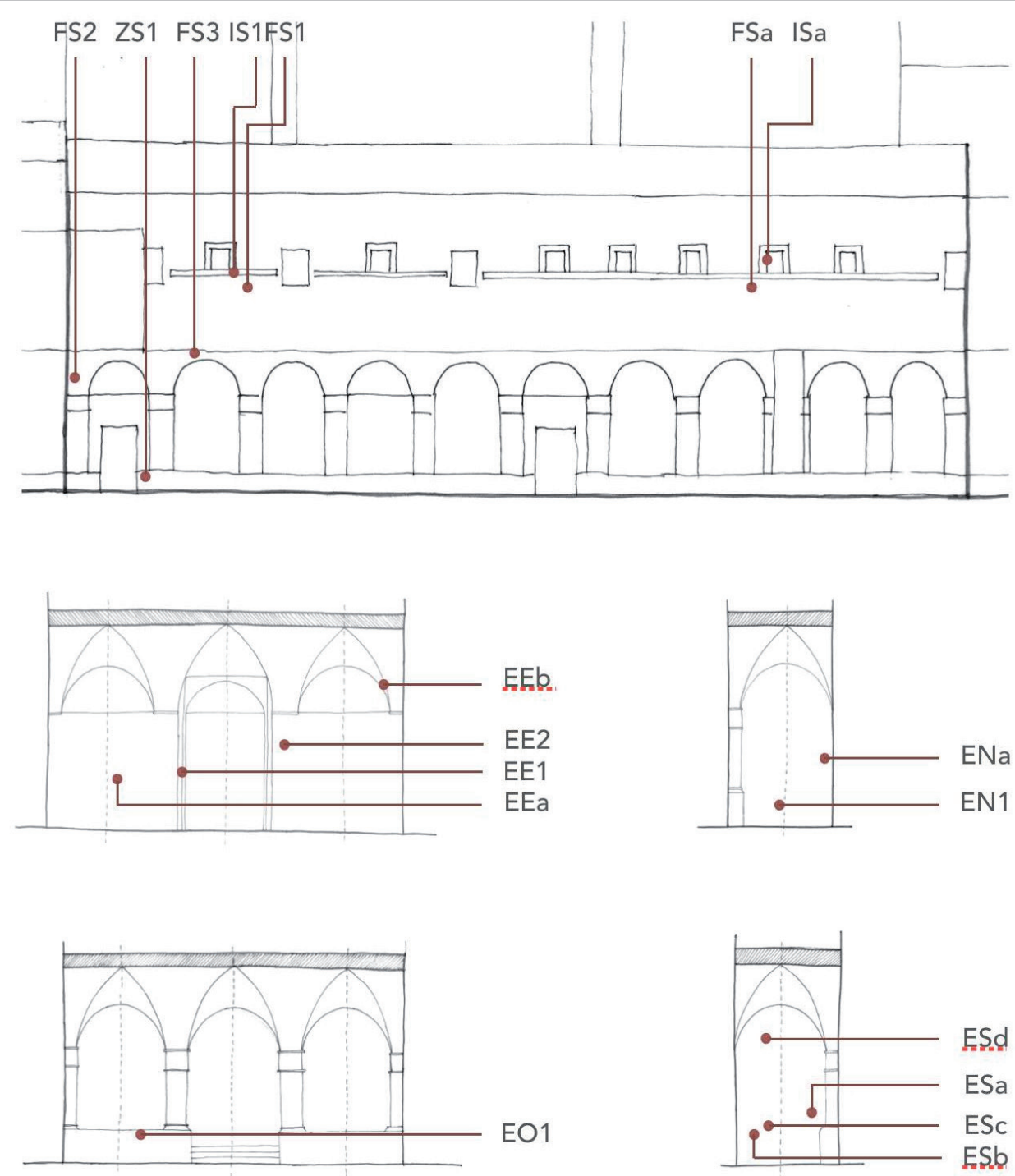

EO1

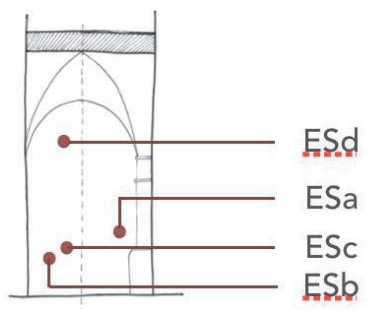

Figura 4.- Alzados del Muro Sur (arriba), y Pórtico de Entrada al Este (en medio, izquierda), al Norte (en medio, derecha), al Oeste (abajo izquierda) y al Este (abajo derecha), y localización de las unidades estratigráficas. Créditos: Isolina Díaz-Ramos.

\section{Escala Munsell}

El sistema de ordenación de color Munsell, ideado por Albert Munsell en 1905 a fin de regular modelos cromáticos, presenta un repertorio de diversos colores sistematizados de manera tridimensional y en progresión en base a su apariencia (Munsell Color Company, 1970). Les otorga tres atributos, como son tono o matiz (hue), valor (value) y croma (chroma).

El tono se basa en cinco nominaciones principales (Hue Symbol) descritas originariamente en inglés: Yellow (Y), Red (R), Green (G), Blue (B) y Purple (P), y cinco intermedias: Yellow-Red (YR), Green-Yellow (GY), Blue-Green (BG), Purple-Blue (PB) y Red-Purple (RP). Estos diez tonos se corresponden con los españoles amarillo, rojo, verde, azul y violeta para los matices principales, y amarillo rojizo, verde amarillento, azul verdoso, violeta azulado y rojo violáceo para sus derivados [Figura5]

El valor en el sistema Munsell es expresado como la luz o sombra de un color en relación a una escala neutra de grises, graduándose desde un negro puro o absoluto, representado como 0/, hasta llegar al blanco absoluto, referido como 10/.

El croma señala el grado de desviación o separación de un tono determinado con otro gris neutro de similar valor. Es decir, un gris neutro posee una escala que va desde el $/ 0$ para los colores neutros, hasta el /10, /12,/14 y /16 según la saturación de la muestra observada.

Blancos y negros, o colores acromáticos, poseen una tabla anexa al atlas Munsell. Su nomenclatura se expresa desde un negro oscuro neutro N1/ hasta un blanco neutro N9/, y se expresa en porcentajes. 


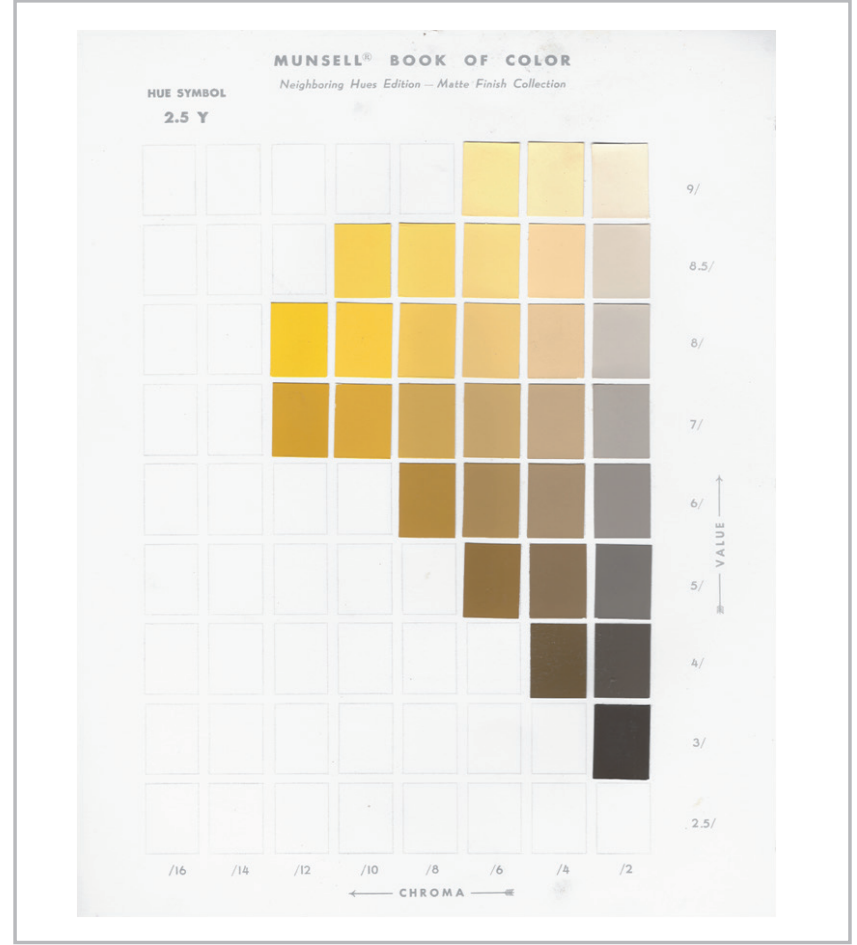

Figura 5.- Carta Munsell correspondiente al tono o matiz 2.5Y en la que se pueden apreciar los atributos de dicho color. Créditos: Isolina Díaz-Ramos.

\section{Registros de color}

Como se ha indicado, en septiembre de 2019 se procedió a la toma de lecturas y concordancias de color de las unidades estratigráficas que ya se hallaban en el Pórtico de Entrada al monumento siguiendo la escala de color Munsell. Fueron registradas cuatro unidades en el muro sur, denominadas en este trabajo ESa, ESb, ESc y ESd. El muro norte, sin embargo, presentaba apenas una secuencia estratigráfica, llamada $\mathrm{ENa}$, mientras que el muro este contenía dos: EEa y EEb.

Las dos unidades estratigráficas presentes en el Muro Sur del claustro fueron denominadas ISa y FSa. Como su nombre indica, se encuentran en la línea de imposta y en el fondo del muro. Sin embargo, no pudieron ser leídas de manera óptima debido al difícil acceso a las mismas, pese a disponer de medios auxiliares [Figura 6].

Los registros cromáticos efectuados por la autora que no requirieron de raspados superficiales debido a que el mal estado de conservación de los muros permitía la lectura de sus estratos a simple vista, fueron denominados EN1, EE1, EE2 y EO1 para aquellos situados en el Pórtico de Entrada. Los realizados en el Muro Sur fueron designados FS2, FS3
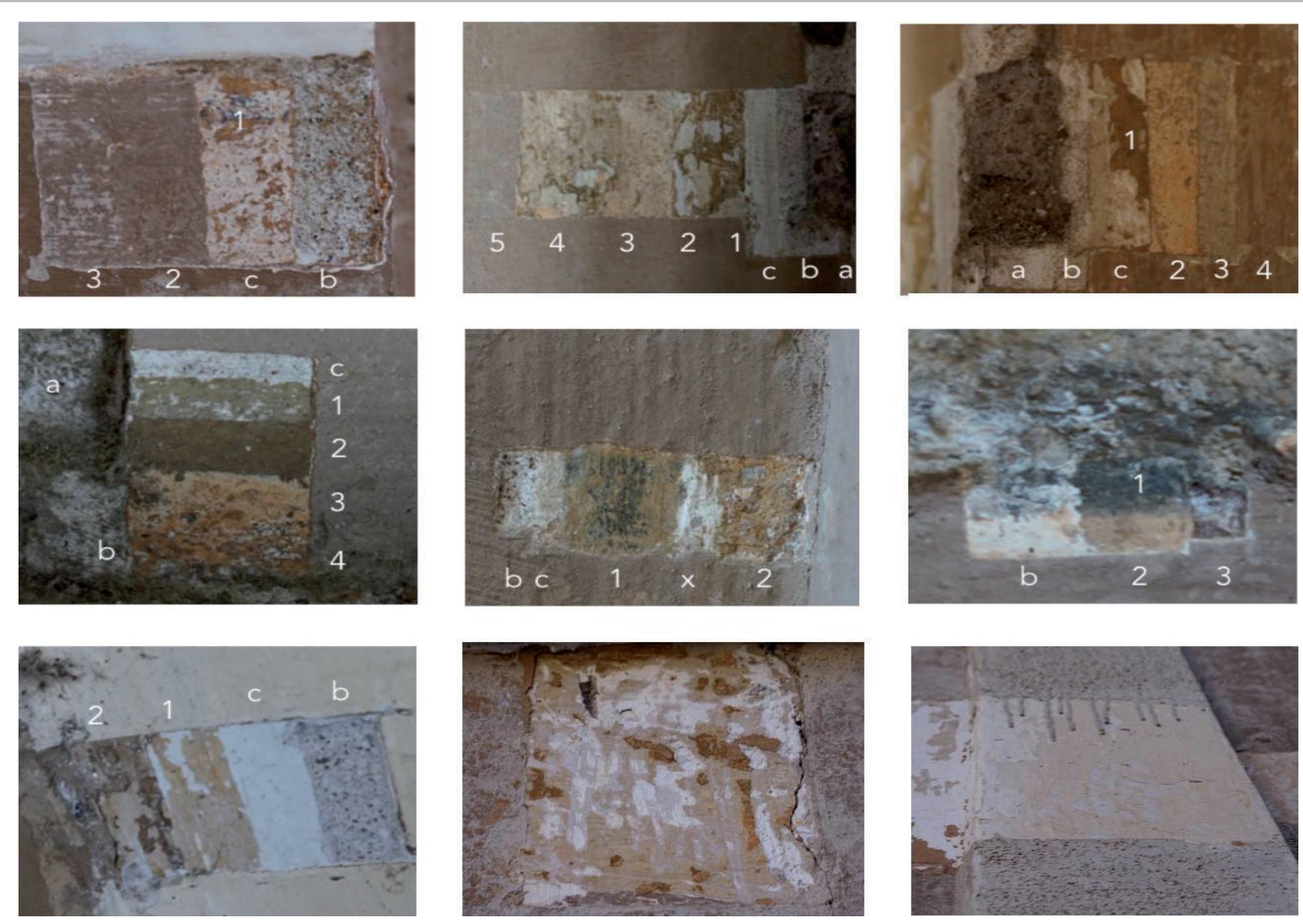

Figura 6.- Documentación de las unidades estratigráficas existentes en el claustro. De izquierda a derecha y de arriba a abajo: ESa, ESb, ESc, ESd, ENa, EEa, EEB, FSa e ISa. En ellas, a representa el mortero grueso, $b$ el mortero fino, y c la capa preparatoria, seguidos de las numeradas secuencias de color o pintura de base. Créditos: Isolina Díaz-Ramos. 

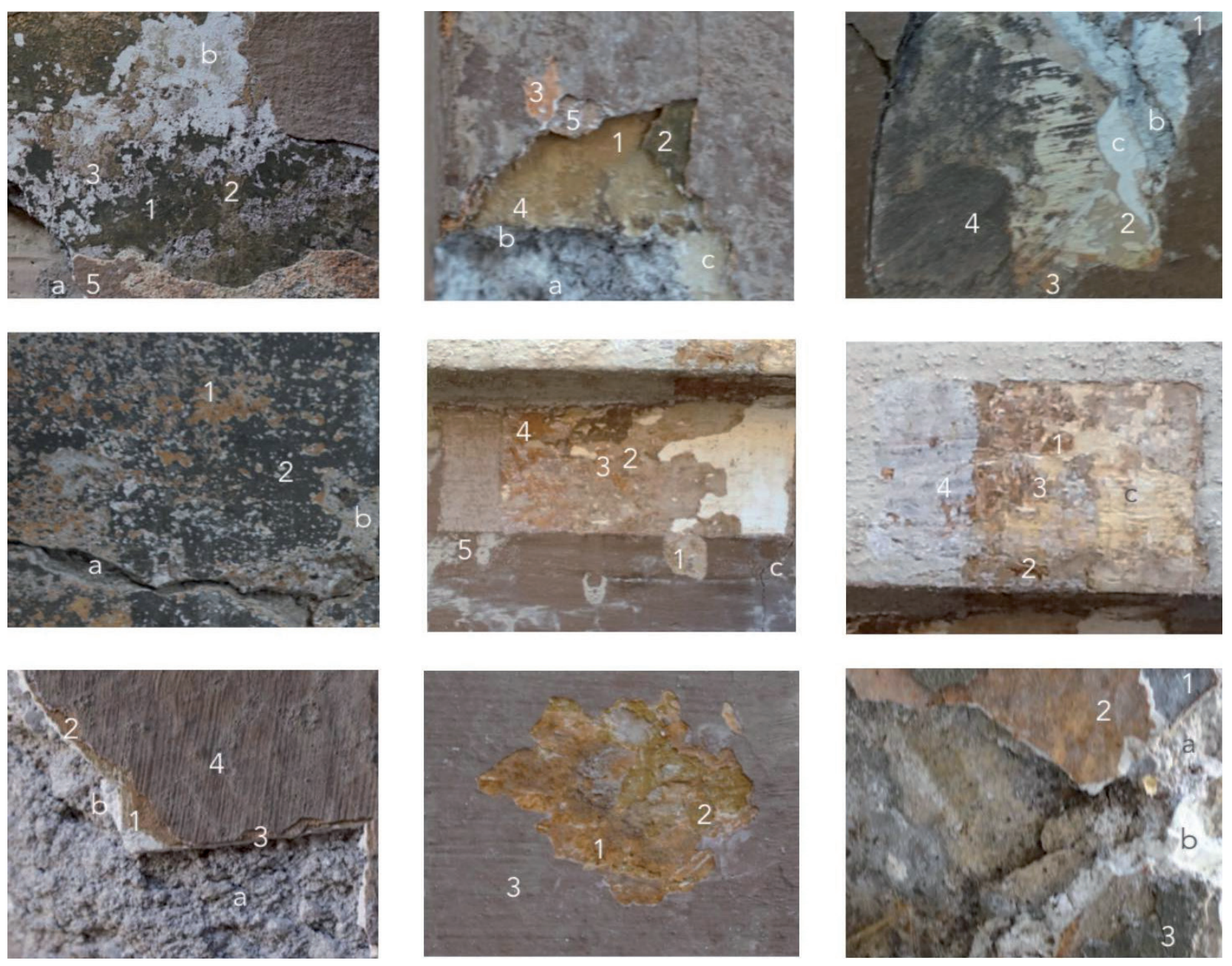

Figura 7.- Documentación de las unidades estratigráficas realizadas por la autora. De izquierda a derecha y de arriba a abajo: EN1, EE1, EE2, EO1, FS1, IS1, FS2, FS3 y ZS1. Créditos: Isolina Díaz-Ramos.

y ZS1. A su vez, a fin de completar la toma de datos, en el Muro Sur se hizo necesaria la realización de dos nuevas unidades estratigráficas, siendo denominadas FS1 e IS1 [Figura 7].

\section{Resultados}

En la zona correspondiente al Pórtico de Entrada se practicaron un total de once lecturas de color [Tabla 1]. En líneas generales, tras el análisis visual de sus unidades estratigráficas, se puede observar en la mayoría de ellas un mortero aplicado en dos capas, en cuyo fondo se aprecia una base de grano grueso (ESb, ESc, ESd, EN1, EE1, EO1 y ZS1). Le sigue un mortero de acabado con gran cantidad de carga fina (ESa, ESb, ESc, ESd, ENa, EEb, EN1, EE1, EE2 y EO1). Sin embargo, en los pilares que dan acceso a esta Galería Oriental, se puede advertir a simple vista la estructura de ladrillo sobre la que reposa un mortero grueso de hasta $10 \mathrm{~mm}$ de espesor. Este mortero es totalmente diferente al documentado en el interior de Pórtico de Entrada, siendo con toda probabilidad aplicado en una intervención reciente.
En el Muro Sur se tomaron cinco lecturas, localizadas en el zócalo, línea de imposta y fondo del muro (FS1, FS2, FS3, IS1, ZS1). En estas zonas no se ha hendido en las paredes, de ahí la ausencia de resultados referidos a los morteros. Abundan en el fondo del muro y el zócalo capas de un material blanquecino, producto de intervenciones posteriores acometidas en el claustro (FS2 y ZS1). De igual modo, la línea de imposta (IS1) no presenta originariamente la textura que se aprecia hoy en día, siendo su fondo totalmente liso [Tabla 2].

Tanto en el Pórtico de Entrada como en el Muro Sur, se ha documentado una capa preparatoria de tonalidad blanca sobre el mortero fino, aplicada con el objetivo de construir una superficie delgada sobre la que adherir la pintura (ESb, ESd, EEb y FS1). Esta pintura base posee una textura muy lisa, con un cierto brillo y transparencia. En determinadas muestras (ESa, ESc, ENa, EEa, EE1, EE2 e IS1), el tono de la capa de preparación tiende hacia al amarillo o el rojo, ofreciendo valores en la escala de 9/ o incluso 8 / de la tabla Munsell. Estos resultados, relacionados con el blanco absoluto, se han incorporado igualmente en la línea de la tabla denominada 'Pintura base'. 
Tabla 1.- Registro de lecturas del Pórtico de Entrada. Los valores señalados con asterisco pertenecen a la Neutral Value Grey Scale de Munsell.

\begin{tabular}{|c|c|c|c|c|c|c|c|c|c|c|c|}
\hline & ESa & ESb & ESC & ESd & $\mathrm{ENa}$ & EEa & EEb & EN1 & EE1 & EE2 & EO1 \\
\hline $\begin{array}{l}\text { Mortero } \\
\text { grueso }\end{array}$ & & $\mathrm{x}$ & $x$ & $x$ & & & & $x$ & $\mathrm{x}$ & & $\mathrm{x}$ \\
\hline Mortero fino & $\mathrm{x}$ & $\mathrm{x}$ & $x$ & $\mathrm{x}$ & $x$ & & $x$ & $x$ & $x$ & $x$ & $x$ \\
\hline Pintura base & 10YR $9 / 2$ & ${ }^{*} \mathrm{~N} 9.5 / 90.0 \% \mathrm{R}$ & 10YR 8/1 & $\mathrm{x}$ & $2.5 Y 9 / 2$ & 10YR $9 / 2$ & ${ }^{*} \mathrm{~N} 9.5 / 90.0 \% \mathrm{R}$ & & 10YR 9/4 & $5 Y 9 / 1$ & \\
\hline Estrato 1 & $5 Y R 6 / 8$ & $2.5 Y 5 / 4$ & $2.5 \mathrm{Y} 6 / 8$ & $2.5 Y 6 / 4$ & $2.5 Y 4 / 2$ & $5 Y 3 / 2$ & $2.5 Y 8 / 4$ & $5 Y 4 / 2$ & $7.5 Y R 5 / 6$ & 10YR $8 / 4$ & $\begin{array}{l}7.5 Y R \\
5 / 6\end{array}$ \\
\hline Estrato 2 & $7.5 Y R 6 / 2$ & $7.5 Y R$ $9 / 4$ & 5YR $8 / 4$ & $2.5 Y 4 / 4$ & $\begin{array}{l}\text { Capa } \\
\text { gruesa } \\
\text { blanca }\end{array}$ & $5 Y R 7 / 4$ & 10YR $4 / 4$ & $7.5 Y R 6 / 6$ & $5 Y 4 / 4$ & $2.5 Y 6 / 4$ & $5 Y 4 / 2$ \\
\hline Estrato 3 & $5 Y R 8 / 2$ & 5YR 8/8 & 7.5 YR $7 / 2$ & $\begin{array}{l}7.5 Y R \\
8 / 4\end{array}$ & $\begin{array}{l}7.5 Y R \\
6 / 10\end{array}$ & $10 R 4 / 2$ & & 10YR $9 / 2$ & $5 Y R 7 / 4$ & $5 Y R 7 / 8$ & \\
\hline Estrato 4 & 5YR 5/6 & $7.5 Y R 6 / 2$ & 5YR $5 / 4$ & 5YR 6/8 & & & & 7.5YR $8 / 6$ & $2.5 Y R 6 / 8$ & 10R $6 / 1$ & \\
\hline Estrato 5 & & $7.5 Y R$ 6/4 & & & & & & 5YR 5/2 & $5 Y R 6 / 4$ & & \\
\hline
\end{tabular}

Tabla 2.- Registro de lecturas en el Muro Sur. Los valores señalados con asterisco pertenecen a la Neutral Value Grey Scale de Munsell.

\begin{tabular}{|c|c|c|c|c|c|}
\hline & FS1 & IS1 & FS3 & FS2 & ZS1 \\
\hline Mortero grueso & & & & & $x$ \\
\hline Mortero fino & & & & Material blanquecino & Material blanquecino \\
\hline Pintura base & $x$ & 10YR 9/4 & & & \\
\hline Estrato 1 & $2.5 Y 7 / 4$ & 5YR $5 / 8$ & $2.5 Y 5 / 6$ & $2.5 Y 8 / 4$ & $10 R 5 / 2$ \\
\hline Estrato 2 & 10YR 6/8 & 7.5YR 7/4 & $5 Y R 7 / 10$ & 7.5YR 5/4 & 7.5YR 5/4 \\
\hline Estrato 3 & 7.5YR 4/2 & 10YR 5/4 & $7.5 \mathrm{R} 6 / 2$ & 7.5YR $8 / 6$ & $5 Y 5 / 2$ \\
\hline Estrato 4 & 7.5YR 6/10 & ${ }^{*} \mathrm{~N} 9.5 / 90.0 \% \mathrm{R}$ & & 7.5YR 5/4 & \\
\hline Estrato 5 & 5YR $8 / 2$ & & & & \\
\hline
\end{tabular}

Sobre la capa preparatoria, han sido hallados en total cuatro estratos históricos hasta llegar a la quinta o superficie actual, compuesta de dos capas de pintura a imitación de un estucado veneciano: un marrón rojizo de fondo, con lectura entre los valores 5 YR $5 / 2$ y 5 YR $5 / 6$. Este quinto estrato varía según se presente más o menos limpio en superficie, como ocurre en las muestras ESb y FS1, donde ha ofrecido unas lecturas en concordancia con 7.5 YR 6/4 y 5 YR $8 / 2$ respectivamente. Sobre esta base de color, se aplicó como terminación un blanco.

El primer estrato de color sobre la pintura base que respondería a la coloración original del claustro, ha dado como resultado tanto en el Pórtico de Entrada como en el Muro Sur, una variedad de valores y cromas correspondientes al tono 2.5Y. La consecución de matices dentro de la misma línea vertical del valor se corresponde con un amarillo verdoso (2.5Y 5/4, 6/4, 7/4 y $8 / 4)$. Este último tono, el 2.5 Y $8 / 4$ aparece duplicado en las muestras EEb y FS3. Otros valores y cromas próximos dentro de la paleta $2.5 \mathrm{Y}$ han sido $5 / 6,6 / 8$, y 4/2, tonos muy similares a los mencionados con anterioridad (ver Figura 5).

Otras lecturas coincidentes se corresponden al tono 7.5YR 5/6, localizado en las muestras EO1 y EE1. Como sus nombres indican, son registros efectuados sin raspados previos en el muro, por lo que este matiz vuelve a aparecer en estratos posteriores de las unidades estratigráficas ya presentes en el Pórtico de Entrada.

De manera general, y tras el análisis de los resultados coincidentes de todos los estratos pictóricos, se puede establecer que la coloración del claustro del Templete de Bramante se ha cubierto a lo largo de su historia de tonos situados en la siguiente gama: 2 Y, 2.5 Y, 2.5YR, 5YR, 7.5YR, 10YR y $10 R$. Es decir, variaciones de una paleta de tonos cálidos compuesta por amarillos, amarillos rojizos, y rojos [Figura 8]. 


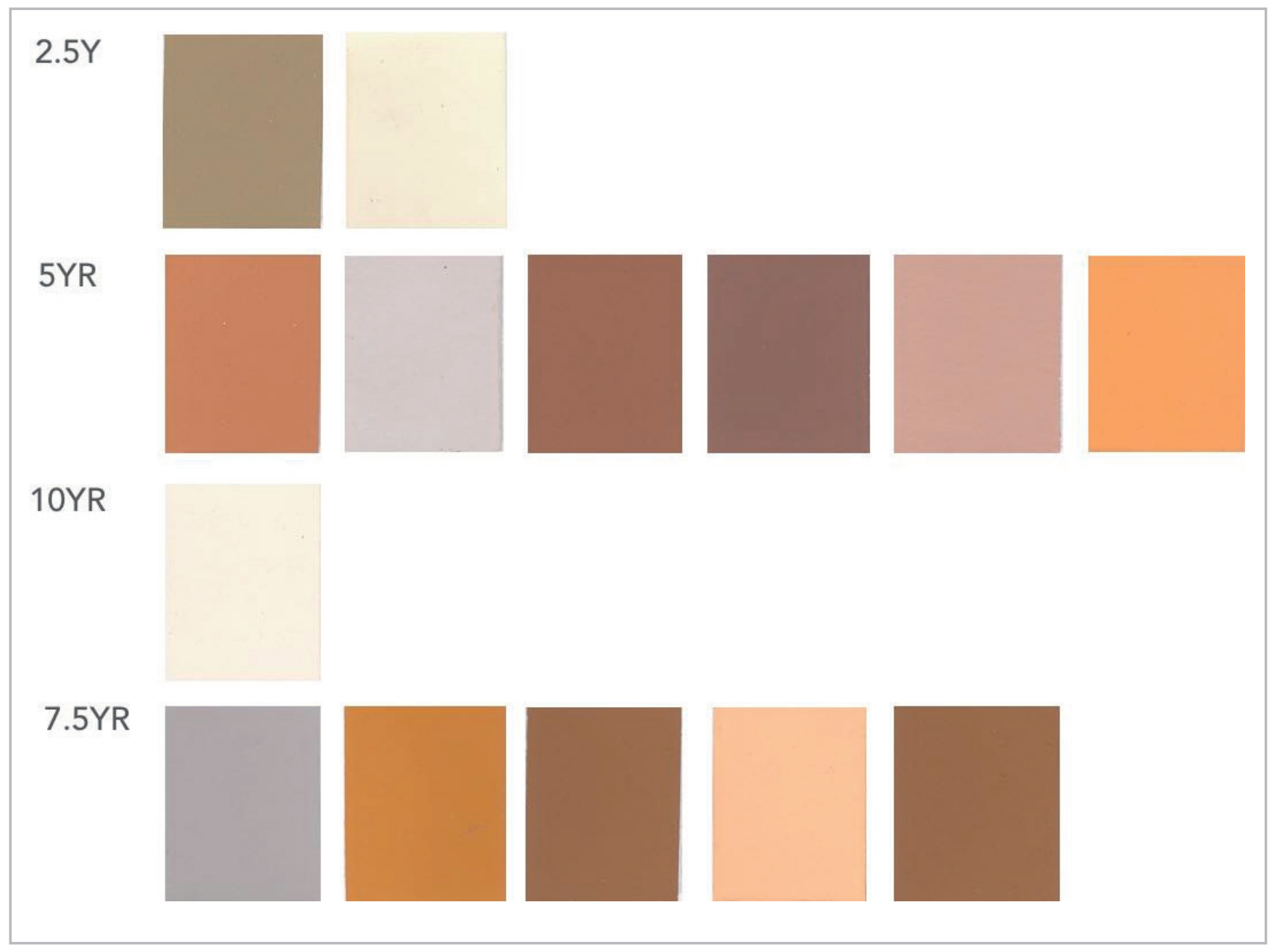

Figura 8.- Estratos de color que se registran al menos dos veces en el. en el claustro del Templete.

\section{Discusión}

Uno de los objetivos de este trabajo era el de documentar y analizar la imagen original y las sucesivas históricas del claustro del Templete de Bramante a fin de ofrecer una base sobre la que continuar poniendo en valor el estudio y conservación de los acabados arquitectónicos.

Aunque se trata de un pequeño estudio efectuado en determinadas zonas del claustro, los resultados obtenidos pueden generalizarse a una sucesión de estratos compuestos de un mortero de grano grueso seguido por otro de grano fino donde reposa una capa de preparación sobre la que se han dispuesto cuatro tonos. Los matices predominantes son amarillos, ocres y rojos, siendo la primera capa de color de tonalidad amarilla, y la última quinta capa o actual rojiza.

Esta investigación se ha realizado sólo en el Muro Sur y el Pórtico de Entrada al claustro, por lo que podría ser interesante incluir la totalidad del perímetro mural del patio del Templete, a fin de sumar a las lecturas efectuadas otras que, en mayor número, completarían la comprensión del conjunto y facilitarían la homogeneización de resultados.
Trabajos similares apoyados en descripciones cualitativas de color como los mencionados de Armenta (2014) y Brino (1987, 1985a y 1985b) difieren de éste en cuanto que no presentan análisis descriptivos de los morteros que acompañan los estratos pictóricos, siendo necesario incluir en el estudio de acabados arquitectónicos los morteros que habrán de influenciar a la coloración posterior.

Aunque numerosas lecturas han coincidido, el lugar que los tonos o matices ocupan en los estratos pictóricos es variable. La falta de uniformidad en el orden de los estratos, no ha ofrecido unos resultados homogéneos en cuanto a la lectura de las unidades cromáticas como se esperaba al inicio de este estudio. Aún así, se pueden apreciar escasas diferencias tonales en una misma línea de estratos, lo que indica una aproximación tonal dentro de los datos registrados, y que sería interesante ajustar en posteriores trabajos.

Por todo ello, las mediciones obtenidas son aproximadas, dado que el resultado de una lectura depende del lugar donde se halle expuesta la muestra (sombra-luz) y del momento del día (hora de la lectura). Esto se ha tenido en cuenta a la hora de efectuar las lecturas, tomándose en 
una misma franja horaria para evitar, en la medida de lo posible, estas variaciones. De igual modo, el que el Muro Sur estuviese expuesto al aire libre, y el Pórtico de Entrada a cubierto, ha podido influir en la variación tonal de los resultados obtenidos, como se ha visto en el apartado anterior con las muestras ESb y FS1.

Por otro lado, la lectura de los estratos ha sido dificultosa en determinadas zonas debido a la adhesión de las capas pictóricas entre ellas, por lo que no se ha podido establecer con precisión el orden de las mismas. La lectura derivada de EO1 presenta sólo dos estratos de color, por lo que es posible que visualmente no se apreciaran estratos inferiores a estas capas, a diferencia de EE1, que pese a realizarse sin raspados sí ha permitido una clara lectura de las cuatro capas cromáticas, incluyendo la quinta que se corresponde con la superficie actual.

Como se mencionó en la Introducción, el color es un aspecto fundamental a tener en cuenta en trabajos de documentación y conservación de bienes patrimoniales arquitectónicos, por lo que la aplicación de metodologías para su estudio, basadas en métodos empíricos, analíticos, o la combinación de ambos, resultan muy valiosos en nuestro territorio.

A día de hoy se sabe que los sistemas de ordenación del color basados en coordenadas de valores triestímulos están apoyados en una base fisiológica, por lo que solo pueden ofrecer resultados informativos y subjetivos, útiles para llamar la atención acerca de lo que a simple vista se encuentra semi-oculto en los muros. Por ello, se hace necesario el acompañamiento de estos registros por estudios profundos, ampliados con medios analíticos de laboratorio que ayuden a determinar la coloración de originales históricos (Díaz-Ramos 2020).

Por lo tanto, la importancia de los resultados obtenidos tras el uso del sistema de ordenación del color Munsell reside en la rápida obtención de datos durante los trabajos de campo. Esta documentación permite poner en valor un elemento arquitectónico y estético determinante como es el color, que contribuye además a incrementar el valor patrimonial de autenticidad.

\section{Conclusiones}

Este estudio preliminar ha localizado y traído a la luz los morteros y estratos pictóricos históricos que componen el claustro del Templete de Bramante. Los datos más relevantes se han hallado sobre todo en el Pórtico de Entrada, donde se ha podido apreciar, gracias y pese al mal estado de conservación de la superficie arquitectónica, restos del primer mortero aplicado de forma gruesa en el fondo, seguido de otro de grano más fino, sobre el que reposa una capa de preparación blanquecina. Sobre ella, se ha detectado una variedad de secuencias cromáticas, trasladadas al sistema Munsell y compuestas por cuatro estratos de color que dan paso a la coloración actual, siendo las tonalidades predominantes aquellas compuestas por amarillos, ocres y rojos, ofreciendo estos datos avances en el presente estado del conocimiento del sitio histórico.

Esta investigación ha contribuido, por lo tanto, a la constatación de la existencia de acabados históricos en los muros del claustro de necesaria investigación. Aunque las lecturas realizadas con la tabla Munsell no ofrecen suficientes evidencias acerca del orden y coloración de los estratos pictóricos debido a la no coincidencia en las lecturas, el empleo de otras técnicas de análisis podrá resolver de manera fehaciente este problema.

En este sentido, y siguiendo la metodología de la Architectural Paint Research (APR), se debería continuar con la extracción de un número representativo de muestras en todo el perímetro del claustro a fin de ser analizadas mediante microscopía óptica y otros métodos científicos de análisis. El apoyo en análisis instrumentales permitiría la obtención de datos concluyentes en la determinación de la secuencia de los estratos, composición de los morteros y los pigmentos. La APR de igual modo, contribuye a indagar en el momento histórico en el que fueron aplicados los diferentes originales históricos, determinando de esta manera los gustos estéticos de cada época.

Como conclusión final, este artículo ha puesto en valor los diferentes estratos que se encuentran en los muros del patio del Templete de Bramante, sirviendo como base sobre la que continuar investigando. A partir de aquí, queda abierto un campo a futuras propuestas de conservación de la superficie arquitectónica, así como en los criterios a seguir en la conservación y restauración de los acabados históricos.

\section{Agradecimientos}

La autora desea expresar su agradecimiento a la Directora de la Real Academia de España en Roma (RAER), Doña Ángeles Albert, a la encargada de la biblioteca, Doña Margarita Alonso, así como al resto del personal de la RAER que favoreció el desarrollo de este trabajo.

De igual manera, Doña María Mata Caravaca, gerente de registros y archivos, y Doña Daniela Sauer, jefa bibliotecaria del ICCROM, facilitaron el préstamo de la Tabla Munsell.

\section{Notas}

[1] El término 'acabado histórico' o 'acabado arquitectónico', conocido en inglés como 'architectural finish' se refiere en este trabajo a todo el sistema de revoco, incluyendo tanto los morteros como los estratos pictóricos. El resto de la terminología empleada en este artículo ha sido extraída y viene definida en el glosario EwaGloss, citado en la bibliografía. 
[2] Este estudio fue realizado siguiendo la línea de investigación que la autora se encontraba realizando dentro del marco de las Becas MAEC-AECID de Arte, Educación y Cultura para el curso académico 2018-2019 dentro del programa de becas para la Real Academia de España en Roma.

\section{Referencias}

ARMENTA, C. (2014). Estudio del color: Carrera del Darro de Granada. Granada: Universidad de Granada.

BERMÚDEZ-CORONEL, F.; RUIZ DEL PORTAL, M. (2000). Estudio estratigráfico de enlucidos. PH: Boletín del Instituto Andaluz del Patrimonio Histórico. 33: 133 - 130.

BOSCH, I. (2014). Proyecto básico y de ejecución y estudio de seguridad y salud de las obras de Consolidación y Restauración de la Cubierta de la Iglesia de San Pietro in Montorio en Roma. Roma, Biblioteca de la Real Academia de España en Roma.

BOSCH, I., ROIG, P., et al. (2010). Informe del Estado de Conservación de la Iglesia de San Pietro in Montorio, de Roma. Roma, Biblioteca de la Real Academia de España en Roma.

BOSCH, I., ROIG, P., SALVADOR, N., MARCENAC, V. (2010). "La iglesia de San Pietro in Montorio di Roma. Actuaciones para su permanencia". En: Arché. Publicación del Instituto Universitario de Restauración del Patrimonio de la UPV. 4-5:295-302. http://hdl. handle.net/10251/31105

BRINO, G. (1987). Colore e città. I colori di Torino 1801-1863. Milano: Idea Books Edizioni.

BRINO, G. (1985a). I colori del Piemonte. Contributo alla formazione di una banca dei dati. Torino: Regione Piemonte.

BRINO, G. (1985b). Il piano del colore di Saluzzo. Torino: Gruppo Editoriale forma.

BUENO, A. (1985). Proyecto de Rehabilitación de Diversos Espacios en la RAER. Roma, Biblioteca de la Real Academia de España en Roma.

CANALDA, S. (2019). Francisco de Asís en San Pietro in Montorio. Los frescos de la Real Academia de España. Madrid: Ministerio de Asuntos Exteriores, Unión Europea y Cooperación - AECID.

CANTATORE, F. (2017). II Tempietto di Bramante nel monastero di San Pietro in Montorio. Roma: Edizione Quasar - AECID.

COLLADO, F., MEDINA, V., GARCÍA, A. (2007). Metodología de estudio cromático de acabados arquitectónicos. Aplicación en la ciudad de Granada. Granada: Universidad de Granada.

COLLADO, F., MEDINA, V., HERNÁNDEZ, A. (2004). El estudio cromático como examen previo y medio de control en el proceso de recuperación del color urbano. En: III Congreso del Grupo Español del IIC. Pp. 265-278.
DÍAZ-RAMOS, I. (2020). "El color del patrimonio urbano edificado. Aproximación a su estudio". En: XV Coloquio de Geografía Urbana. Las Palmas: Universidad de Las Palmas de Gran Canaria.

GARCÍA, A., LLOPIS, J., TORRES, A., VILLAPLANA, R. (2012). EI color de Valencia. El centro histórico. Valencia: Excelentísimo Ayuntamiento de Valencia, Generalitat Valenciana.

GARCÍA, A., LLOPIS, J. (2010). Estudio histórico del color del Centro Histórico de Ontiyent. Valencia: Ajuntament d'Ontiyent.

INTERNATIONAL COUNCIL ON MONUMENTS AND SITES (1994). The Nara Document on Authenticity. Nara: UNESCO, ICCROM and ICOMOS.

JIMÉNEZ, A. (2013). "La Academia de España en Roma, antiguo convento de San Pietro in Montorio. Imágenes de su evolución arquitectónica y paisajística". En: EGA Expresión Gráfica Arquitectónica, [S.I.], n. 22, p. 224-233, nov. 2013. ISSN 2254-6103. [Consulta: 23/10/2019] https://doi.org/10.4995/ ega. 2013.1301

MUNSELL COLOR COMPANY (1970). Munsell Book of Color. Baltimore, United States: Munsell Color Company.

PELÁEZ, J. (1992). Memoria Anteproyecto de Plan Director de la Academia de Bellas Artes en Roma. Roma, Biblioteca de la Real Academia de España en Roma.

PELÁEZ, J. (2003). Restauración puntual de Fábricas en el Claustro del Tempietto. Notificaciones oficiales y Estudio de Seguridad y Salud. Roma, Biblioteca de la RAER.

PELÁEZ, J., FRECHILLA, J. (2002a). Anteproyecto de Plan Director RAER. Roma, Biblioteca de la Real Academia de España en Roma.

PELÁEZ, J., FRECHILLA, J. (2002b). Actualización y Ampliación del Plan Director RAER. Roma, Biblioteca de la Real Academia de España en Roma.

PELÁEZ, J., FRECHILLA, J. (2001). Avance de Anteproyecto de Plan Director RAER. Roma, Biblioteca de la Real Academia de España en Roma.

SANCHO RODA, J., CAPPONI, S. (1998). Proyecto de Restauración del Tempietto de Bramante. Roma, Biblioteca de la Real Academia de España en Roma.

SANCHO RODA, J., SANCHEZ BARRIGA, A. (1997-1999). Memoria Final Obras de Emergencia Previas a la Restauración del Tempietto. Roma, Biblioteca de la Real Academia de España en Roma.

WEYER, A., ROIG, P., POP, D., CASSAR, J., ÖZKÖSE, J., SRŠA, I. (2016). EwaGlos. European Illustrated Glossary of conservation terms for wall paintings and architectural surfaces. Petersberg, Germany: Michael Imhof Verlag. 


\section{Autor/es}

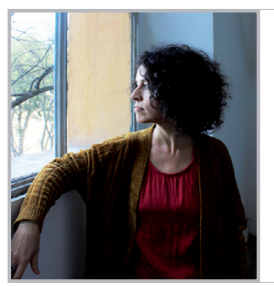

\section{Isolina Díaz-Ramos}

isodiram@gmail.com

Universidad de Las Palmas de Gran Canaria.

Doctora en arquitectura por la Universidad de Las Palmas de Gran Canaria y Conservadora y Restauradora de Obras de Arte por la Universidad Politécnica de Valencia. Desarrolla su investigación en torno a la conservación y restauración de superficies de arquitecturas vernáculas, pintura mural y patrimonio arqueológico. Ha realizado estancias de investigación en la Universidad Moulay Ïsmail de Mequines, Marruecos; Universidad Nacional del Nordeste, Argentina y la Universidad de Alexandria, Egipto. De igual modo, ha recibido diversos premios, como la reciente estancia de investigación en la Fundación Bogliasco, Génova y en la Real Academia de España en Roma.

Artículo enviado el 24/05/2020 Artículo aceptado el 25/08/2020

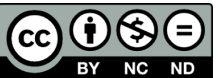

https://doi.org/10.37558/gec.v18i1.772 